\title{
STRUCTURE SHARING PROBLEM AND ITS SOLUTION IN GRAPH UNIFICATION
}

\author{
Kiyoshi KOGURE \\ NTT Basic Research Laboratories \\ 3-1 Morinosato-Wakamiya, Atsugi-shi, Kanagawa, 243-01 Japan \\ kogureQatom.ntt.jp
}

\begin{abstract}
The revised graph unification algorithms presented here are more efficient because they reduce the amount of copying that was necessary because of the assumption that data-structure sharing in inputs occurs only when feature-structure sharing occurs.
\end{abstract}

\section{INTRODUCTION}

Constraint-based linguistic frameworks use logical systems called feature logics (Kasper \& Rounds, 1986; Shicber, 1989; Smolka, 1988), which describe linguistic objects by using logical formulas called feature descriptions that have as their models feature structures or typed feature structures. Shiebcr (1989) argued that if the canonical models of finite formulas of a feature logic were themselves finite, we could use them to compute over instead of theorem-proving over the formulas themselves. This would be advantageous if we had efficient algorithms for manipulating the canonical models.

The most important operation on models-feature structures or typed feature structures - is combining the information two models contain. This operation is traditionally called unification, although re cently it has come to be more suitably called informational union. This unification operation is significant not only theoretically but also practically because the efficiency of systems based on constraintbased formalisms depends on the (typed) feature structure unification and/or feature description unification algorithms they use. ${ }^{1}$ This dependency is especially crucial for monostratal formalisms-that is, formalisms which use only (typed) feature structures such as HPSG (Pollard \& Sag, 1987) and JPSG (Gunji, 1987). ${ }^{2}$

The efficiency of (typed) feature structure unification has been improved by developing algorithms that take as their inputs two directed graphs representing (typed) feature structures, copy all or part of them, and give a directed graph representing the unification result. These algorithms are thus called graph unification. Previous research has identified graph copying as a significant overhead and has attempted to reduce this overhead by lazy copying and structure sharing.

Unification algorithms developed so far, however, including those allowing structure sharing scem to

\footnotetext{
${ }^{1}$ For example, the TASLINK natural language system uses $80 \%$ of the processing time for feature structure unification and other computations required by unification, i.e., feature structure pre-copying (Godden, 1990).

${ }^{2}$ For example, a spoken-style Japanese sentence analy sis system based on HPSG (Kogure, 1989) uses 90\%-98\% of the processing time for feature structure unification.
}

$$
\operatorname{syn}\left[\begin{array}{l}
\text { agree: } \mathrm{X}: \operatorname{agr}\left[\begin{array}{ll}
\text { num }: \text { sg } \\
\text { per: } & \text { 3rd }
\end{array}\right] \\
\text { subj: } \operatorname{syn}[\text { agree: } \mathrm{X}]
\end{array}\right]
$$

Fig. 1: Matrix notation for a typed feature structure.

contradict structure sharing because they assume the two input graphs never share their parts with each other. This "structure sharing" assumption prevents the initial data structures from sharing structures for representing linguistic principles and lexical information even though many lexical items share common information and such initial data structure sharing could significantly reduce the amount of data structures required, thus making natural language systems much more efficient. Furthermore, even if the structure sharing assumption holds initially, unification algorithms allowing structure sharing can yield situations that violate the assumption. The ways in which such unification algorithms are used are therefore restricted and this restriction reduces their efficiency.

This paper proposes a solution to this "structure sharing problem" and provides three algorithms. Section 2 briefly explains typed feature structures, Section 3 defines the structure sharing problem, and Section 4 presents key ideas used in solving this problem and provides three graph unification algorithms that increase the efficiency of feature structure unification in constraint-based natural language processing.

\section{TYPED FEATURE STRUCTURES}

'The concept of typed feature structures augments the concept of feature structures. A typed feature structure consists of a set of feature-value pairs in which each value is a typed feature structure. The set of type symbols is partially ordered by subsumption ordering $\leq_{T}$ and constitutes a lattice in which the greatest element $T$ corresponds to 'no information' and the least element $\perp$ corresponds to 'over-defined' or 'inconsistency.' For any two type symbols $\mathbf{a}, \mathbf{b}$ in this lattice, their least upper bound and greatest lower bound are respectively denoted $\mathbf{a} \vee_{\tau} \mathbf{b}$ and $\mathbf{a} \wedge_{T} \mathbf{b}$.

Typed feature structures are represented in matrix notation as shown in Fig. 1, where syn, agr, sg, and 3rd are type symbols; agree, num, per, and subj are feature symbols; and $X$ is a tag symbol. A featureaddress - that is, a finite (possibly empty) string of feature symbols - is used to specify a feature value of an embedded structure. In Fig. 1, for example, the structure at the feature-address agree num, where ' $\because$ is the concatenation operator, is said to have $\mathrm{sg}$ as its type symbol. The root feature-address is de- 


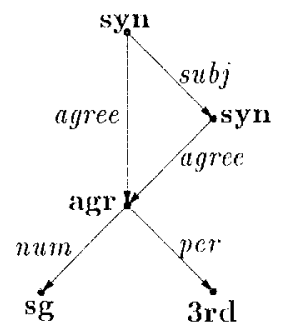

lig. 2: Graph representation of a typed feature structurc.

noted by ' $r$.' 'lo specify token-identity in matrix notation, a tag symbol is used: feature-address values with the same tag symbol are token-identical, and these feature-addresses with the token-identical value are said to corefer. In lig. 1, the feature-inddresses agree and subj ayree corefer.

A typed feature structure is also represented by a rooted, connected, directed graph within which each node corresponds to a typed feature structure and is labeled with a type symbol (and, optionally, a tag symbol) and each arc corresponds to a feature-value pair and is labeled with a feature symbol. Fig. 2 illustrates the graph representation of the typed feature structure whose matrix notation is shown in Fig. 1. In a graph representation, the values at coreferent feature-addresses- that is, token-iclentical values are represented by the same node.

'The set of typed feature structures is also partially ordered by a subsumption ordering that is an extension of the subsumption ordering on the set of type symbols. $A$ typed feature structure $t_{1}$ is loss than or equal to $t_{2}$ (written as $t_{1} \leq_{t} t_{2}$ ) if and only if $t_{1}$ is inconsistent (that is, if it includes the type symbol $\perp$ ) or (i) $t_{1}$ 's type symbol $a_{1}$ is less than or equal to $t_{2}$ 's type symbol a. ( $\left.a_{1} \leq_{\mathcal{T}} a_{2}\right)$; (ii) each feature $f$ of $t_{2}$ cxists in $\ell_{1}$ and has a value $\ell_{2, f}$ such that its comiterpart $t_{1, f}$ is less than or equal to $t_{2, f}$; and (iii) each coreference relation holding in $t_{2}$ also holds in $t_{1}$.

'Ihis subsumption ordering serves as the basis for defining two latitico operations: goneralization (the least upper bound or join) and mification (the greatest lower bound or meet).

Typed feature structures have been formalized in several ways, such as by using $\psi$-types (Ait-Kaci, 1986).

\section{THE STRUCTURE SHARING PIROIBLEM}

\subsection{Graph Unification Algorithms}

The destructive unification algorithm presented by Nit-Kaci is the starting point in increasing the efficiency of graph unification. It is a node-morging process that uses the Union-lind algorithm, which was originally developed for testing finite antomata equivalence (Hopcroft \& Karp, 1971), in a manner very similar to that of the unification algorithm for rational terms (Huet, 1976). Given two root nodes of graphs representing (typed) feature structures, this algorithm simultaneously traverses a pair of input nodes with the same feature-address, putting them

\begin{tabular}{|c|c|}
\hline \multicolumn{2}{|r|}{ node structure } \\
\hline tsymbol & $\langle$ a type symbol $\rangle$ \\
\hline arcs & 〈a set of arc structures \\
\hline generation & 〈an integer \\
\hline forward & $\bar{N} I I \mid\langle a$ node structure $\rangle$ \\
\hline copy & $\begin{aligned} & N I I \mid\langle\text { a node structure }\rangle \\
& \mid \text { (a copydep structure } \\
&\end{aligned}$ \\
\hline \multicolumn{2}{|r|}{ arc structure } \\
\hline label & 〈a feature symbol $\rangle$ \\
\hline value & 〈a node structure \\
\hline \multicolumn{2}{|r|}{ copydep structure } \\
\hline generation & 〈an integer \\
\hline deps & 〈a set of node and arc pairs) \\
\hline
\end{tabular}

l'ig. 3: Data structures for nondestructive unification and IIJNG unification.

into a new and larger coreference class, and then returns the morged graph.

Since the destructive unification process modifies its input graphs, they must first be copied if their contents are to be preserved. Nondeterminism in parsing, for example, requires the preservation of graph structures not only for initial graphs representing lexical entries and phrase structure rules but also for those representing well-formed intermediate structures. Although the overhead for this copying is significant, it is impossible to represent a resultant unified graph without creating any now structures. Unnecessary copying, though, must be identified and minimized. Wroblewski (1987) defined two kinds of unnecessary copying-over-copying (copying structures not neoded to represent resultant graphs) and early-copying (copying structures even though unification fails)- -but this account is flawed because the resultant graph is assumed to consist only of newly created structures cven if parts of the inputs that are not changed during unification could be shared with the resultant graph. A more efficient unification algorithm would avoid this redundant copying (copying structures that can be shared by the input and resultant graphs) (Kogure, 1990). 'To distinguish structure sharing at the implementation level from that at the logical level (that is, coreference relations between feature-addresses), the former is called data-structure sharing and the latter is called foature-structure sharing ('ornabechi, 1992).

The key approaches to reducing the amount of structures copied are lazy copying and data-structure sharing. Vor lazy copying, Karttumen (1986) proposed a reversible unification that saves the original contents of the inputs into preallocated areas inmediately before destructive modification, copies the resultant graph if necessary, and then restores the original contents by undoing all the changes made during unification. Wroblewski (1987), on the other hand, proposed a nondestructive unification with incremental copying. Given two graphs, Wroblewski's algorithm simultaneously traverses each pair of input nodes with the same feature-address and creates a common copy of the input nodes. 'The nondestructive unification 
algorithm for typed feature structures uses the data structures shown in Fig. 3. ${ }^{3}$ The algorithm connects an input node and its copy node with a copy linkthat is, it sets the copy node as the input's copy field value. 'The link is meaningful during only one unification process and thus enables nondestructive modification. ${ }^{4}$ Using an idea similar to Karttunen's, Tomabechi (1991) proposed a quasi-destructive unification that uses node structures with fields for keeping update information that survives only during the unification process. ${ }^{5}$

Unification algorithms allowing data-structure sharing (DSS unification algorithms) are based on two approaches: the Boyer and Moore approach, which was originally developed for term unification in theorem-proving (Boyer \& Moore, 1972) and was adopted by Pereira (1985); and the lazy copying suggested by Karttunen and Kay (1985). Recent lazy copying unification algorithms are based on Wroblewski's or Tomabechi's schema: Godden (1990) proposed a unification algorithm that uses active data structures, Kogure (1990) proposed a lazy incremental copy graph (LING) unification that uses dependency-directed copying, and Emele (1991) proposed a lazy-incremental copying (LIC) unification that uses chronological dereference. 'These algorithms are based on Wroblewski's algorithm, and Tomabechi (1992) has proposed a data-structure-sharing version of his quasi-destructive unification.

\subsection{The Structure Sharing Problem}

The graph unification algorithms mentioned so far-perhaps all those developed so far - assume that data structure sharing between two input structures occurs only when feature-structure sharing occurs between feature-addresses they represent. This "structure sharing" assumption prevents data-structure sharing between initial data structures for representing linguistic principles and lexical information even though many lexical items share common information. For cxample, many lexical items in a traditional syntactic categories such as noun, intransitive verb, transitive verb, and so on share most of their syntactic information and differ in their semantic aspects such as semantic sortal restriction. Such initial data-structure sharing could significantly reduce the amount of data structures required and could therefore reduce pageswapping and garbage-collection and make natural language processing systems much more efficient.

Furthermore, even if the structure sharing assumption holds initially, applying a DSS unification algorithm in natural language processing such as parsing and generation can give rise to situations that violate the assumption. Consider, for example, JPSG-

\footnotetext{
${ }^{9}$ For the nondestructive unification algorithm, the node structure takes as its copy field value either NTL or a node structure only.

In this algorithm each unification process has an integer as its process identifier and each node created in a process has the identifier as its generation field value. $A$ copy link is meaningful only if its destination node has the current process identifier. Such a node is called 'current.'

${ }^{5}$ r The technique used to control the lifetime of update data is the same as that of Wroblewski's algorithm.
}

based parsing. There are only a few phrase structure rules in this framework and the Complement-Head Construction rule of the form ' $\mathrm{M} \rightarrow \mathrm{C}$ H' is applied very frequently. For instance, consider constructing a structure of the form $\left[\mathrm{VP}_{2} \mathrm{NP}_{2}\left[\mathrm{VP}_{1} \mathrm{NP}_{1} \mathrm{~V}\right]\right]$. When the rule is applied, the typed feature structure for the rule is unified with the structure resulting from embedding the typed feature structure for $\mathrm{NP}_{1}$ at the feature-address for the complement daughter in the rule (e.g., dtrs $\cdot c d t r$ ), and the unification result is then unified with the structure resulting from embedding the typed feature structure for $V$ at the feature-address for the head daughter. Because not every substructure of the structure for the rule always changed during such a unification process, there may be some substructures shared by the structure for the rule and the structure for VP $P_{1}$. Thus, when constructing $\mathrm{VP}_{2}$ there may be unexpected and undesired data-structure sharing between the structures.

Let me illustrate what happens in such cases by using a simple example. Suppose that we use the nondestructive unification algorithm or one of its datastructure sharing versions, the LING or LIC algorithm. The nondestructive and IING unification algorithms use the data structures shown in Fig. 3, and the IIC algorithm uses the same data structures except that its node structure has no forward field. Consider unification of the typed feature structures $t_{1}$ and $t_{2}$ shown in Fig. 4(a). Suppose that $t_{1}$ and $t_{2}$ are respectively represented by the directed graphs in Fig. 4(b) whose root nodes are labeled by tag symbols $X_{0}$ and $X_{4}$. 'That is, $t_{1}$ 's substructure at feature-address $f_{2}$ and $t_{2}$ 's substructure at $f_{1}$ are represented by the same data structure while featurcstructure sharing does not hold between them, and $t_{1}$ 's substructure at $f_{3}$ and $t_{2}$ 's substructure at $f_{4}$ are represented by the same data structure while featurestructure sharing does not hold between them. Wach of the algorithms simultaneously traverses a pair of input nodes with the same feature-address both of the inputs have from the root feature-address to leaf feature-addresses, makes a commor copy of them to represent the unification result of that featureaddress, and connects the input and output nodes with copy links. For any feature-address that only one of the inputs has, the nondestructive unification algorithm copies the subgraph whose root is the node for that feature-address and adds the copied subgraph to the output structure, whereas the LING and IIC algorithms make the node shared by the input and output structures. In the case shown in Fig. 4(b) the root nodes of the inputs nodes with the tag symbols $X_{0}$ and $X_{4}$-are first treated by creating a common copy of them (i.e., the output node with $Y_{0}$ ), connecting the input and output nodes with copy links, and setting $\mathbf{b}_{0}=\mathbf{a}_{0} \wedge_{\tau} \mathbf{a}_{4}$ as the copy's tsymbolvalue. Then the input nodes' arc structures are treated. Suppose that the pair of $f_{1}$ arcs is treated first. After the input nodes at feature-address $f_{1}$ are treated in the same manner as the root nodes, the pair of $f_{2}$ arcs is treated. In this case, $t_{1}$ 's node at $f_{2}$ (labeled $X_{2}$ ) already has a copy link because the node is also used as $t_{2}$ 's node at $f_{1}$ so that the destination node of the link is used as this feature-address's output node. Af- 


$$
\begin{array}{r}
t_{1}: a_{0}\left[\begin{array}{ll}
f_{1}: & a_{1} \\
f_{2}: & a_{2} \\
f_{3}: & a_{3}
\end{array}\right], \\
t_{2}: a_{4}\left[\begin{array}{ll}
f_{1}: & a_{2} \\
f_{2}: & a_{b} \\
f_{4}: & a_{3}
\end{array}\right] .
\end{array}
$$

(a) Itput typed feature structures.

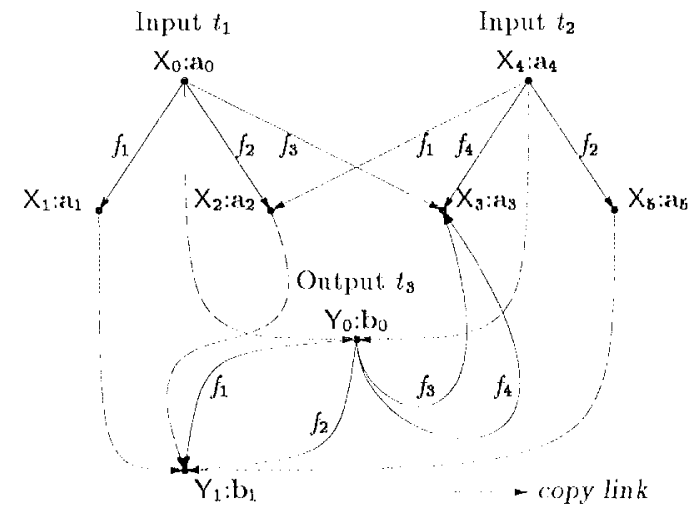

(b) Snapshot of incremental graph mification allowing datia-structure sharing.

$$
\begin{aligned}
t_{3}: & \mathbf{b}_{0}\left[\begin{array}{ll}
f_{1}: & Y_{1}: b_{1} \\
f_{2}: & Y_{1} \\
f_{3}: & x_{3}: a_{3} \\
f_{4}: & x_{3}
\end{array}\right], \\
t_{1} \wedge_{t} t_{2}: & b_{0}\left[\begin{array}{ll}
f_{1}: & b_{2} \\
f_{2}: & b_{3} \\
f_{3}: & n_{3} \\
f_{4}: & n_{3}
\end{array}\right]
\end{aligned}
$$

where

$$
\begin{aligned}
& b_{0}=a_{0} \wedge_{T} a_{4}, \\
& b_{1}=a_{1} \wedge_{T} a_{2} \wedge_{7} a_{b}, \\
& b_{2}=a_{1} \wedge_{T} a_{2}, \\
& b_{3}=a_{2} \wedge_{T} a_{6} .
\end{aligned}
$$

(c) Wrong graph unification output $\left(t_{3}\right)$ and the cor rect unification of the inputis $\left(t_{1} \wedge_{1} t_{2}\right)$.

lig. 4: An example of incortect graph unification.

ter the common label ares are treated, unique label ares are treated. 'The nondestructive unification algorithm copies $t_{1}$ 's $f_{3}$ and $t_{2}$ 's $f_{4}$ arcs and axlds them to the output root node, whereas the IING and TIC algorithms make the input and output structures share their destination nodes. F'inally, the JING and IAC algorithms obtain graph $t_{3}$, represented in matrix notation in l'ig. 4(c) just over the correct result.

The nondestructive unification algorithm obtains the same typed feature structure. 'lhe reversible and the quasi-destructive unification algorithms are also unable to obtain the correct result for this example because these algorithms cannot represent two update nodes by using a single node. 'Thus, none of the efficient unification algorithms developed recently obtains the correct results for such a case. Avoiding such wrong unification results requires undesirable copy- ing. We can, for example, avoid getting the wrong result by interleaving the application of any non-DSS unification algorithm between applications of a DSS unification algorithm, but such bypassing requires two unification programs and reduces the efliciency gain of JSS unification. 'This preclusion of useful datastructure sharing is referred to here as the "structure sharing" problem.

It has been shown that, all the IOSS unification algorithms mentioned above are subject to this problem even if the structure sharing assumption holds initially. Non-I)SS unification algorithms are also subject to the problem because their inputs are created by applying not only the unification operation but also operations such as embedding and extraction, in most implementations of which data-structure shar. ing occurs between their input and output structures. liven non-DSS unification algorithms must therefore take such inputs into account, and this requires nu. desirable copying.

\section{A SOLUTION TO 'THE STRUCTURE SHARING PROBLEM}

\subsection{Kry Ideas}

'The exanple in Section 3 suggests that the structure sharing problem has two sources, which concern not only the incremental copying approach but also other appronches. The first source is the way of recording update information. In the incremental copying approach, this corresponds to the way of copying structures. 'That is, while calculating $l_{1} \wedge_{t} t_{2}$ the incremental copying process does not distinguish between the copies created as the substructures of the left input $t_{1}$ and the copies created as the substructures of the right input $t_{2}$. As a result, a copy node of $t_{1}$ 's node at feature-address $p$ can be used as a copy node of $t 2$ 's node at a feature-address, and vice vorsa. In lig. 4(b), for example, the copy of $t_{2}$ 's node at $f_{2}$ is wrongly used as the copy of $t_{1}$ 's node at $f_{1}$. 'This causes unexpected and wrong data-structure sharing in the resultant graph and this in turn canses unexpected ind wrong feature-structure sharing in the resultant (typed) feature structure. In other approaches, such as the quasi-destructive approach, the source of the structure sharing problem is that each node structure has fields for keeping information on only two typed feature structures -one for the original and one for the result - whercas fields for kecping information on three typed feature structures are needed- - one for the original and one for each of the two results.

One way to solve this problem is therefore to mako each node keep information on three typed feature structures: in the incremental copying approach cach node must have two copy fields, and in the quasi. destruclive approach cach node must have two sets of ficlds for updates.

The second source of the strueture sharing problem is the method of clata-structure sharing between input and output structures. Unexpected and wrong data-structure sharing may result if a node shared by the left and right inputs is used as part of the left input, intended to be shared between the left input and output, at the sarre time it is used as part of the right input, intended to bo shared between the right input 


\begin{tabular}{|c|c|}
\hline \multicolumn{2}{|r|}{ node structure } \\
\hline tsymbol & 〈a type symbol \\
\hline arcs & $\langle$ a set of arc structures \\
\hline generation & $\langle$ an integer $\rangle$ \\
\hline forward & $N I L \mid\langle$ a node structure $\rangle$ \\
\hline lcopy & $N I L \mid\langle$ a node structure $\rangle$ \\
\hline$r \operatorname{cop} y$ & $N L \mid\langle$ a node structure $\rangle$ \\
\hline
\end{tabular}

Fig. 5: The node structure for the revised nondestructive unification.

and output. In Fig. 4(b), for example, $t_{1}$ 's node at feature-address $f_{3}$ is shared as $t_{3}$ 's node at the same feature-address, and the same node as $t_{2}$ 's node at $f_{4}$ is shared as $t_{3}$ 's node at the same feature-address.

This problem can be solved easily by keeping information on data-stucture sharing status; that is, by adding to the node structure a new field for this purpose and using it thus: when a unification algorithm makes a node shared (for example, between the left input and output), it records this information on the node; later when the algorithm attempts to make the node shared, it does this only if this data-structure sharing is between the left input and output.

\subsection{Algorithms}

This section first describes a non-DSS unification algorithm that discards the structure sharing assumption and thus permits initial data-structure sharing, and then it describes two JSSS unification algorithms. Revised Nondestructive Unification

This algorithm uses, instead of the node structure shown in Fig. 3, the node structure in Fig. 5. 'I'hat is, the algorithm uses two kinds of copy links: lcopy for the left input and rcopy for the right input.

The revised nondestructive unification procedure for typed feature structures is shown in Figs. 6 and 7 . Given two root nodes of directed graphs, the top-level procedure Unify assigns a new unification process identifier, generation, and invokes Unify_Aux. 'This procedure first dereferences both input nodes. This dereference process differs from the original one in that it follows up forward and lcopy links for the left input node and forward and reopy links for the right input node. This revised dereference process eliminates the first source of the structure-sharing problem. Then Unify A ux calculates the meet of the type symbol. If the meet is $\perp$, which means inconsistency, it finishes by returning $L$. Otherwise Unify_Aux obtains the output node and sets the meet as its tsymbol value. 'The output node is created only when neither input node is current; otherwise the output node is a current input node. 'Then Unify_Aux treats arcs. 'I'his procedure assumes the existence of two procedures: Shared_Arc_Pairs and Complement_Arcs. 'The former gives two lists of arcs each of which contains ares whose labels exist in both input nodes with the same arc label order; the latter gives one list of arcs whose labels are unique to the first input node. For each arc pair obtained by Shared_Ar__Pairs, Unify_A $A x$ applies itself recursively to the value pair. And for each arc obtained by Complement_Arcs, it copies its value.

Jet us compare the newly introduced cost and the

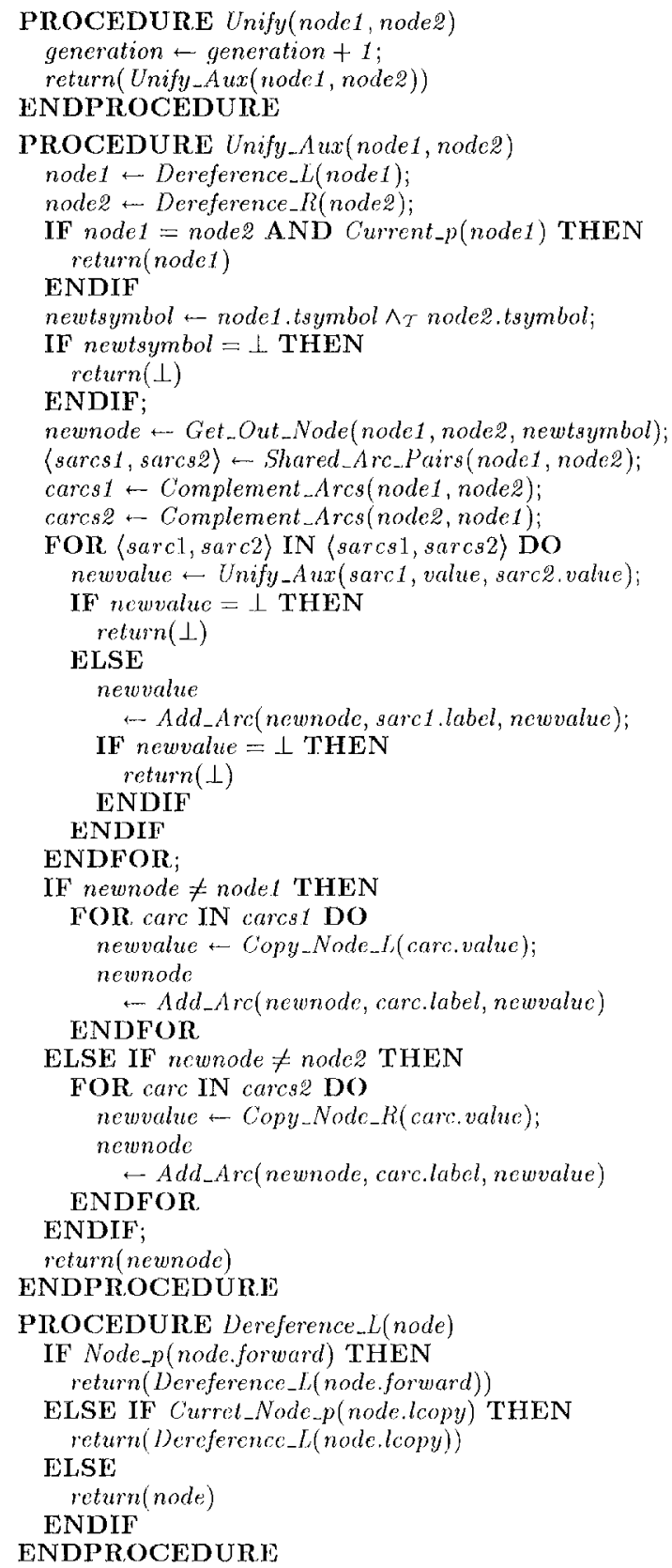

Fig. 6: 'The revised nondestructive unification procedure (1).

effect of this revision. This revised version differs from the original in that it uses two dereference procedures that are the same as the original dereference procedure except that they use different fields. Thus, on the one hand, the overhead introduced to this revision is only the use of one additional ficld of the node structure. On the other hand, although this revised version does not introduce new data-structure sharing, it can safely treat data-structure sharing in ini- 


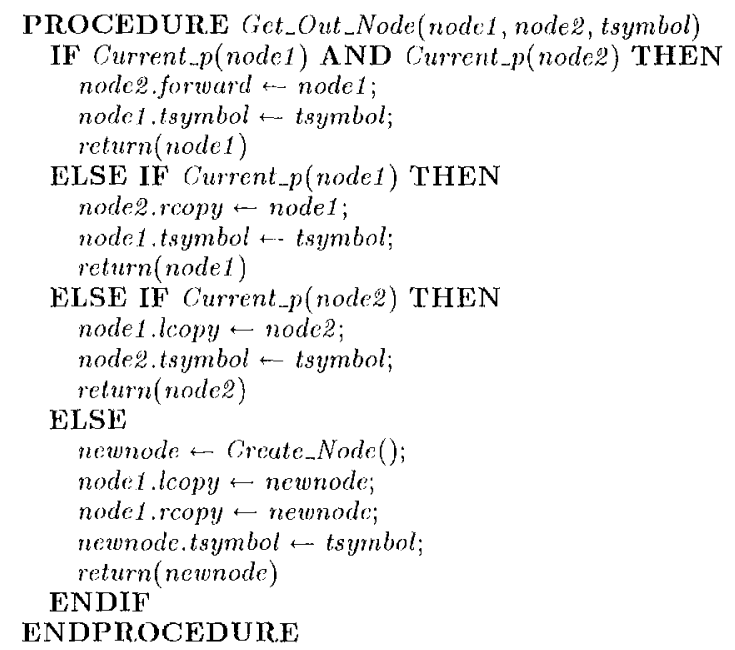

Fig. 7: 'The revised nondestructive unification procedure (2).

tial datia structures. This can significantly reduce the amount of initial data structures required for linguistic descriptions, especially for lexical descriptions, and thus reduce garbage-collection and page-swapping.

\section{Revised LING Unification}

LING unification is based on nondestructive unification and uses copy-dependency information to implement data-structure sharing. For a unique label arc, instead of its value being copied, the value itself is used as the output value and copy-dependency relations are recorded to provide for later modification of shared structures. This algorithm uses a revised Copy_Node procedure that takes as its input two node structures (node 1 and node.2) and one arc structure, arcl where nodel is the node to be copied. The structure arc1 is an are to nodel, and nodel is an ancestor node of node 1 - that is, the node from which are1 departs -and the revised procedure is as follows: (i) if nodet' (the dereference result of nodet) is current, then Copy-Node returns node1' to indicate that the ancestor noded must be copied immediately; otherwise, (ii) Copy_Ares is applied to nodet' and if it returns several arc copics, Copy_Node creates n now copy node and then adds to the now node the are copies and ares of nodel' that are not copied, and returns the new node to indicate the ancestor node having to be copied immediately; otherwise, (iii) Copy_Node registors the copy-dependency between the nodet' and the ancestor node node?.that is, it adds the pair consisting of the ancestor nodo nodeg and the arc are 1 into the copy field of node 1 and returns NII, to indicate that the ancestor must not be copied immediately. ${ }^{6}$ When a new copy of a node is needed later, this algorithm will copy struc-

\footnotetext{
${ }^{\theta}$ Tin the IIN 1 unificaiton algorithm, a node structure's copy field is used to keep cither copy information or copydependency information. Whon the ficld keeps copydependency information, its value is a copydep structure consisting of an integer generation field-and a set of
}

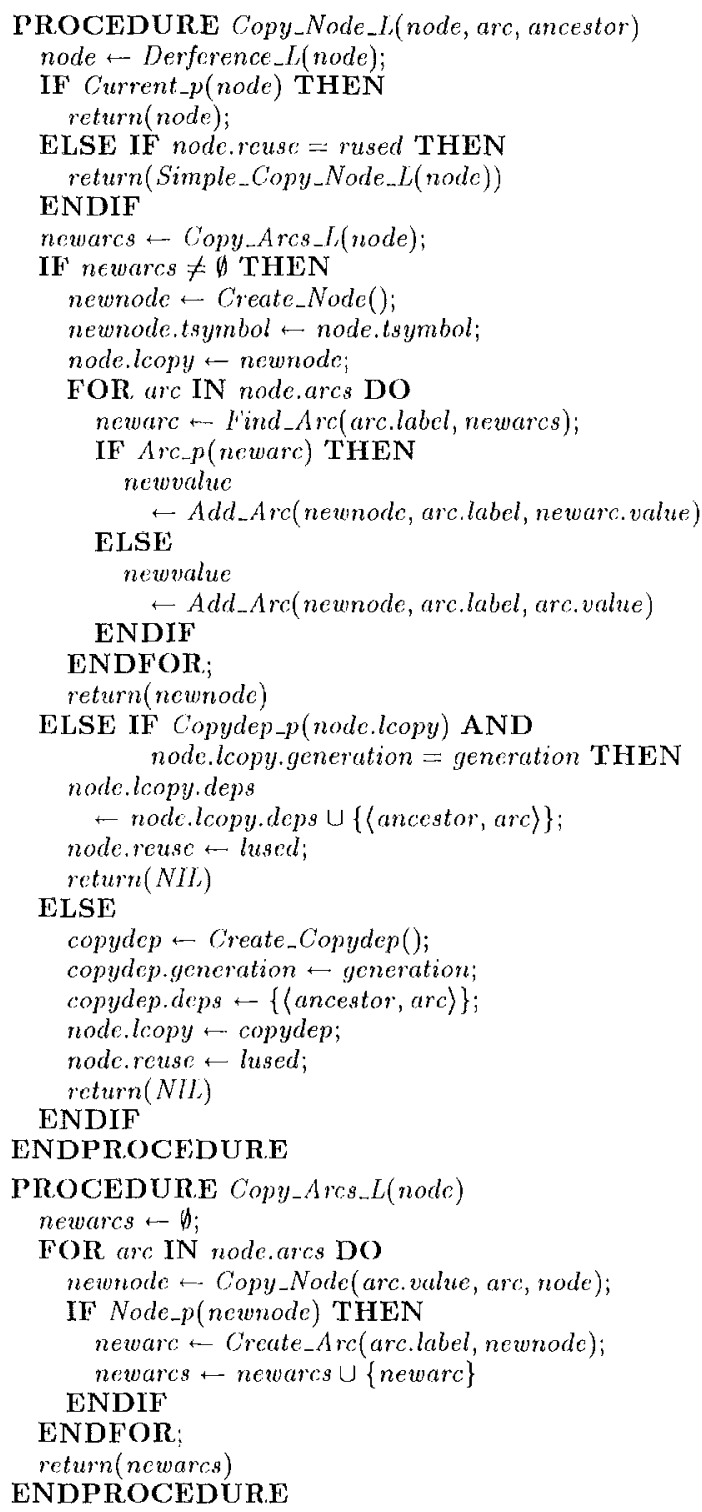

l'ig. 8: 'The new revised Copy_Node procedure.

tures by using the copy-dependency information in its copy field (in the revised Get_Out_Node procedure for: the JING unification). It substitutes arcs with newly copied nodes for existing ares. Thus the antecedent nodes are also copied.

The revised LING unification is based on the revised nondestructive unification and uses a nodestruc ture consisting of the ficlds in the node structure shown in Fig, 5 and a new field reuse for indicat-

node and are pairs - deps field (sec Fig. 3). The technique used to control the lifetime of copy-dependency information is the same as that of copy information. That is, the deps field value is meaningful only when the generation value is equal to the unification process identifier. 
ing data-structure sharing status. When the top-level unification procedure is invoked, it sets two new symbols to the two variables lused and rused. That a node structure has as its reuse field value the lused value means that it is used as part of the left input, and that it has as its reuse value the rused value means that it is used as part of the right input. The revised LING unification uses two new revised Copy_Node procelures, Copy Node_l (shown in Fig. 8) and the analogous preocedurc $\mathrm{Copy}_{-} \mathrm{Node}_{-} R$. These procedures are respectively used to treat the left and right inputs and they differ from the corresponding original procedure in two places. First, instead of step (i) above, if node1' (the dereference result of node1) is current, Copy_Node_L (or Copy_Node_R) returns node1' to indicate that the ancestor, node 2 , must be copied immediately. But if nodc1' has as its reuse field value the rused (or lused) value, it creates a copy of the whole subgraph whose root is noder' and returns the copied structure also to indicate that the ancestor node must be copied immediately. Second, in step (iii), they register data-structure sharing status-that is, they set the lused (or ruscd) value to the reuse field of noder' as well as register copy-dependency information. 'This revised LING unification ensures safety in data-structure sharing.

Again let us compare the newly introduced computational costs and the effect of this revision. The nowly introduced costs are the additional cost of the revised dereference procedures (which is the same as in the previous one) and the cost of checking reuse status. The former cost is small, as shown in the discussion of the previous algorithm, and the latter cost is also small. 'These costs are thus not significant relative to the efficiency gain obtained by this revision.

\section{Revised Quasi-Destructive Unification}

The structure-sharing version of quasi-destructive unification keeps upelate information in the ficld meaningfiul only during the unification. After a successful unification is obtained, this algorithm copies the unification result and attempts data-structure sharing. 'This algorithm can be revised to cnsure safety in data-structure sharing by using a node structure including two sets of lields for update information and one reuse field and by checking node reuse status while copying.

\section{CONCLUSION}

The graph unification algorithms described in this paper increase the efficiency of feature structure unification by discarding the assumption that data-structure sharing between two input structures occurs only when the feature-structure sharing occurs between the feature-addresses they represcnt. All graph unification algorithms proposed so far make this assumption and are therefore required to copy all or part of their input structures when there is a possibility of violating it. 'This copying reduces their efficiency. 'This paper analyzed this problem and points out key ideas for solving it. Revised procedures for nondestructive unification, LING unification, and quasi-destructive unification have been developed. 'These algorithms make the use of feature structures in constraint-based natural language procossing much more efficient. 'The. key ideas in this paper can also be used to make the incremental graph generalization algorithm (Kogure, 1993) more efficient.

\section{ACKNOWLEDGMENTS}

I thank Akira Shimazu, Mikio Nakano, and other colleagues in the Dialogue Understanding Group at the NT' Basic Research Laboratories for their oncouragement and thought-provoking discussions.

\section{REFERENCES}

Ait-Kaci, H. (1986). An Algebraic Semantics Approach to the Fffective Resolution of Type Fquations. 7 . of Theor. Comp. Sci., 45, 293 351.

Boyer, R. S., \& Moore, J. S. (1972). The Sharing of Struc:ture in 'Theorem-Proving Programs. In Meltzer, B. \& Michie, D. (Eds.), Machine Intelligence Vol. 7 , cliap. 6, pp. 101-116. Edinburgh University Press.

Emele, M. (1991). Unification with Lazy Non-Redundant Copying. In Proc. of the 29th ACL, pp. 325-330.

Godden, K. (1990). Iazy Unification. In Proc. of the 28th $A C L$, pp. $180-187$.

Gunji, 'L'. (1987). Japanese Plrase Structure Grammar. Reidel.

Ilopcroft, J. E., \& Karp, R. M. (1971). An Algorithm for Testing the Fquivalence of l'inite Automata. Tech. Rep. T'R-71-114, Dept. of Comp. Sci., Cornell University.

Huet, G. (1976). Résolution d'Lquations dans des Langages d'Ordre $1,2, \ldots, \omega$. Ph.D. thesis, Université de Paris VII

Karttunen, T. (1986). D-PATR-- A Development Linvironment for Unification-Based Grammars. 'Iech. Rep. CSLI-86-61, CSII.

Karttunen, I.., \& Kay, M. (1985). Structure Sharing Representation with Binary Trees. In Proc. of the $23 \mathrm{rd}$ ACI, pp. 133-136.

Kasper, R. 'I., \& Rounds, W. C. (1986). A Jogical Semantics for Veature Structure. In Proce of the 24th ACL.

Kogure, K. (1989). Parsing Japanese Spoken Sentences based on HPSG. In Proc. of the Int. Workshop on Parging Technologies, pp. 132-141.

Kogure, K. (1990). Strategic Lazy Incremental Copy Graph Unification. In Proc. of the 13 th COLING Vol. 2, pp. 223-228

Kogure, K. (1993). Typed lieature Structure Generalization by Incremental Graph Copying. In 'I'rost, H (Ld.), leature Formalisms and Linguistic Ambiguity, pp. 139-158. Ellis Horwood.

Pereira, F. C. N. (1985). Structure Sharing Representation for Unification-Based Formalisms. Th Proc. of the 23rd $A C L$, pp. $137 \cdots 144$.

Pollard, C., \& Sag, I. (1987). An Information-Baged Syntax and Semantics-Volume 1: Fundamentals. CSLI Iecture Notes No. 13. CSII.

Shicber, S. M. (1989). Constraint-Based Grammar Formalisms--Parsing and Type Inference for Natural and Computer Languages. Ph.D. thesis, Stanford University.

Smolka, G. (1988). A licature Logic with Subsorts. III,OG 33, IBM Deutschland.

Tomabechi, H. (1991). Quasi-Destructive Graph Unification. In Proc of the $29 t h A C H$, pp. 315-322.

'lomabechi, HI. (1992). Quasi-Destructive Graph Unification with Structure-Sharing. In Proc. of the $1 / 4$ th COLING, pp. $440 \cdots 446$.

Wroblewski, D. A. (1987). Nondestructive Graph Unification. In Proc. of the 6th $A A A I$, pp. 582-587. 